\title{
ORIGINAL ARTICLE Updated cost-effectiveness analysis of supplemental glutamine for parenteral nutrition of intensive-care patients
}

\author{
L Pradelli $^{1}$, M Povero ${ }^{1}$, M Muscaritoli $^{2}$ and M Eandi $^{3}$
}

BACKGROUND/OBJECTIVES: Intravenous (i.v.) glutamine supplementation of parenteral nutrition (PN) can improve clinical outcomes, reduce mortality and infection rates and shorten the length of hospital and/or intensive care unit (ICU) stays compared with standard PN. This study is a pharmacoeconomic analysis to determine whether i.v. glutamine supplementation of PN remains both a highly favourable and cost-effective option for Italian ICU patients.

SUBJECTS/METHODS: A previously published discrete event simulation model was updated by incorporating the most up-to-date and clinically relevant efficacy data (a clinically realistic subgroup analysis from a published meta-analysis), recent cost data from the Italian health-care system and the latest epidemiology data from a large Italian ICU database (covering 230 Italian ICUs and more than 77000 patients). Sensitivity analyses were performed to test the robustness of the results.

RESULTS: Parenteral glutamine supplementation can significantly improve ICU efficiency in Italy, as the additional cost of supplemented treatment is more than completely offset by cost savings in hospital care. Supplementation was more cost-effective (cost-effectiveness ratio (CER) $=€ 35165$ per patient discharged alive) than standard, non-supplemented PN (CER=€40 156 per patient discharged alive), and it resulted in mean cost savings of $€ 4991$ per patient discharged alive or $€ 1047$ per patient admitted to the hospital. Sensitivity analyses confirmed the robustness of these results.

CONCLUSIONS: Alanyl-glutamine supplementation of PN is a clinically and economically attractive strategy for ICU patients in Italy and may be applicable to selected ICU patient populations in other countries.

European Journal of Clinical Nutrition (2015) 69, 546-551; doi:10.1038/ejcn.2014.255; published online 3 December 2014

\section{INTRODUCTION}

Glutamine is one of the 20 common amino acids, and thus it is a very important organic compound. It is also the most abundant free amino acid in the human body. Although endogenous glutamine production is adequate in normal healthy people, glutamine depletion is associated with certain critical illnesses. It is said to become 'conditionally essential' as the body's requirements for glutamine can exceed the individual's ability to produce it, and thus endogenous stores of glutamine are depleted rapidly. This dangerous scenario is demonstrated by studies showing that low plasma glutamine levels in patients admitted to intensive care units (ICUs) are an independent predictor of premature death. ${ }^{1,2}$ Typically, the body's glutamine levels will be most compromised for patients in hypercatabolic and/or hypermetabolic states (e.g., acute pancreatitis, severe burns, sepsis or following major surgical procedures), who thus may benefit most from an exogenous source of glutamine.

Practical problems with the use of free glutamine (e.g., instability and poor solubility) have prevented its widespread use in the routine clinical setting. However, in recent years these problems have been overcome by the use of glutamine dipeptides (e.g., alanyl-glutamine; Dipeptiven). Alanyl-glutamine is highly soluble, and it is stable in solution, allowing storage at room temperature and heat sterilisation. ${ }^{3}$ After its administration, the dipeptide is rapidly split into glutamine and alanine, which then flow as nutrients into their respective body pools and are metabolised according to the body's needs. ${ }^{3,4}$ Clinical benefits of intravenous (i.v.) glutamine supplementation of standard parenteral nutrition (PN) for critically ill patients have included reductions in mortality rates, fewer infectious complications and reduced length of ICU/hospital stay. ${ }^{5}$

The observed clinical benefits of i.v. glutamine supplementation at an adequate dose in patients most likely to benefit has prompted pharmacoeconomic cost-effectiveness studies. Modelling is one cost-effectiveness analysis approach that is used frequently. It combines different types of data (e.g., clinical and financial) within a logical framework that represents the underlying data, thus allowing the cost-effectiveness of an intervention to be determined under conditions of uncertainty. The costeffectiveness of i.v. glutamine supplementation has previously been investigated, and the results were highly favourable: net cost savings were achieved plus the costs of parenteral glutamine supplementation were offset completely. ${ }^{6}$ Net-saving treatments (i.e., better quality of health care for less cost) are exceptional among pharmacoeconomic evaluations.

The current study is an update of previously published data. ${ }^{6}$ The model requires updating, as substantial new efficacy data have been published in the form a meta-analysis. ${ }^{5}$ This metaanalysis included a clinically realistic subgroup analysis based on adequate doses of parenteral glutamine $(>0.2 \mathrm{~g} / \mathrm{kg}$ body weight per day), as recommended by the guidelines. ${ }^{7,8}$ These doses were associated with reduced mortality rates, infection rates and shorter length of hospital stay for ICU patients, and they form the basis of efficacy data used for the current model. ${ }^{5}$ In this article, we will combine the most up-to-date and clinically relevant efficacy data, the most recent cost data and the latest

${ }^{1}$ AdRes Health Economics and Outcomes Research, Turin, Italy; ${ }^{2}$ Department of Internal Medicine, Università La Sapienza, Rome, Italy and ${ }^{3}$ Department of Pharmacology, Università degli Studi di Torino, Torino, Italy. Correspondence: Dr L Pradelli, Adres Health Economics and Outcomes Research, Piazza Carlo Emanuele II, 19, Turin 10123, Italy. E-mail: I.pradelli@adreshe.com 
epidemiology data from a large Italian ICU database to determine whether i.v. glutamine supplementation remains a highly favourable cost-effective option in total PN therapy for intensive care patients.

\section{MATERIALS AND METHODS}

The evaluation was based on a decision-analytic event simulation pharmacoeconomic model. The model combines (i) baseline outcomes from the Italian ICU patient population, (ii) partially re-elaborated efficacy data from a meta-analysis of published randomised clinical trials and (iii) national (Italian) cost data. The following text describes the model structure, baseline event rates and underlying assumptions, efficacy data sources and unit costs included in the modelling.

\section{Model structure}

The model structure used was essentially that described previously by Pradelli et al. ${ }^{6}$ TreeAge Pro 2009 software (TreeAge Software Inc., Williamstown, MA, USA) was used to design a discrete event simulation, which is a type of decision model (other types are decision trees and Markov models). Decision models are used to help decide whether treatment A or treatment B is preferable for a particular type of patient on the basis of two dimensions: costs and health effects. In a discrete event simulation, the experience of individuals is modelled over time in terms of the events that occur and the consequences of those events. A discrete event simulation is more practical than Markov models when timing and chronology of events is important. Moreover, discrete event simulation models are analysed as patient-level simulations, and this can add accuracy to the analysis. In brief, this model was used to compare the assignment of ICU patients with standard PN or PN supplemented with i.v. glutamine. In the simulation, every patient started in the ICU, where they may (or may not) develop a new nosocomial infection. In either case, the patient admitted to the ICU faced three alternative possibilities: (i) death in the ICU, (ii) recovery and transfer to a general ward or (iii) recovery and discharge home. For those transferred to a general ward, there were two possibilities: (i) recovery and discharge or (ii) death (Figure 1). Consistent with this modelling technique, time was not discretised in cycles, but it was handled as time-to-event; the time that patients spent in each state was sampled from distributions fitted to data reported in the Progetto Margherita (Table 1).

\section{Patient population: data sources}

The Progetto Margherita report was used as the ICU population data source for estimating baseline outcome rates (death rates, infection rates and length of hospital/ICU stay) and transition probabilities (probability of changing from one health state to another health state within a given period). ${ }^{9}$ Elaborated data are reported in Table 1, which also details the choice of distributions allocated to each parameter in the model. The Progetto Margherita report used covers data from 2010, which is an update on the 2007 data used in the previous model..$^{10}$ It is an annual publication on behalf of the Gruppo Italiano per la Valutazione degli Interventi in Terapia Intensiva (GIVITI), and it includes data collected in 230 Italian ICUs, from a total of over 77000 patients, covering over half of all ICUs operating in Italy. This well-established project has been used as a data resource for observational investigations, including pharmacoeconomic studies. ${ }^{11-15}$

The time horizon used corresponded to the hospital stay of the patients. The duration of PN assumed that (i) all patients in the treatment arm received at least one full day of PN; (ii) that PN never lasted as long as the hospital stay (ends at least 2 days before the discharge from the hospital to allow for patient stabilisation, excluding the pre-ICU length of stay); and (iii) that for patients who die the duration of PN did not exceed the length of hospital stay.

\section{Clinical efficacy: data sources}

Comparative efficacy data from clinical trials-for standard PN vs supplementation of PN with i.v. glutamine for ICU patients-were taken from a subgroup in the meta-analysis of Bollhalder et al., ${ }^{5}$ and showed clear improvements in relative risk of death, infection and the length of hospital stay (Table 2). This subgroup provided data for a clinically realistic and adequate dose of parenteral glutamine $(>0.2 \mathrm{~g} / \mathrm{kg}$ body weight per day), as recommended by the guidelines, 7,8 and it represented the majority of the patients included in the meta-analysis (54-63\%, depending on the parameter analysed). ${ }^{5}$

The absolute length of stay reduction reported in the meta analysis was converted to a relative duration (relative risk $(R R)=0.91 ; 95 \%$ confidence interval ( $\mathrm{Cl})$ : $0.86-0.96)$ by using the mean hospital length of stay reported by patients given standard PN calculated from Bollhalder et al. ${ }^{5}$ This additional processing is needed, as the absolute reduction estimated by Bollhalder et $a l^{5}$ can be applied only to total length of hospital stay, whereas in our model this is assessed in two stages (length of stay in the ICU and then in the general ward). The relative reduction can thus be applied to both the ICU and the general ward for patients given parenteral glutamine. Moreover, reductions in the length of hospital stay owing to glutamine supplementation are related to the time over which patients are given $\mathrm{PN}$, and thus the efficacy of treatment results are better modelled by relative risk.

\section{Cost assessment: data sources}

The study perspective is that of the health-care provider (i.e., hospitals), and thus only direct costs (e.g., cost of stays in the ICU or ward) rather than

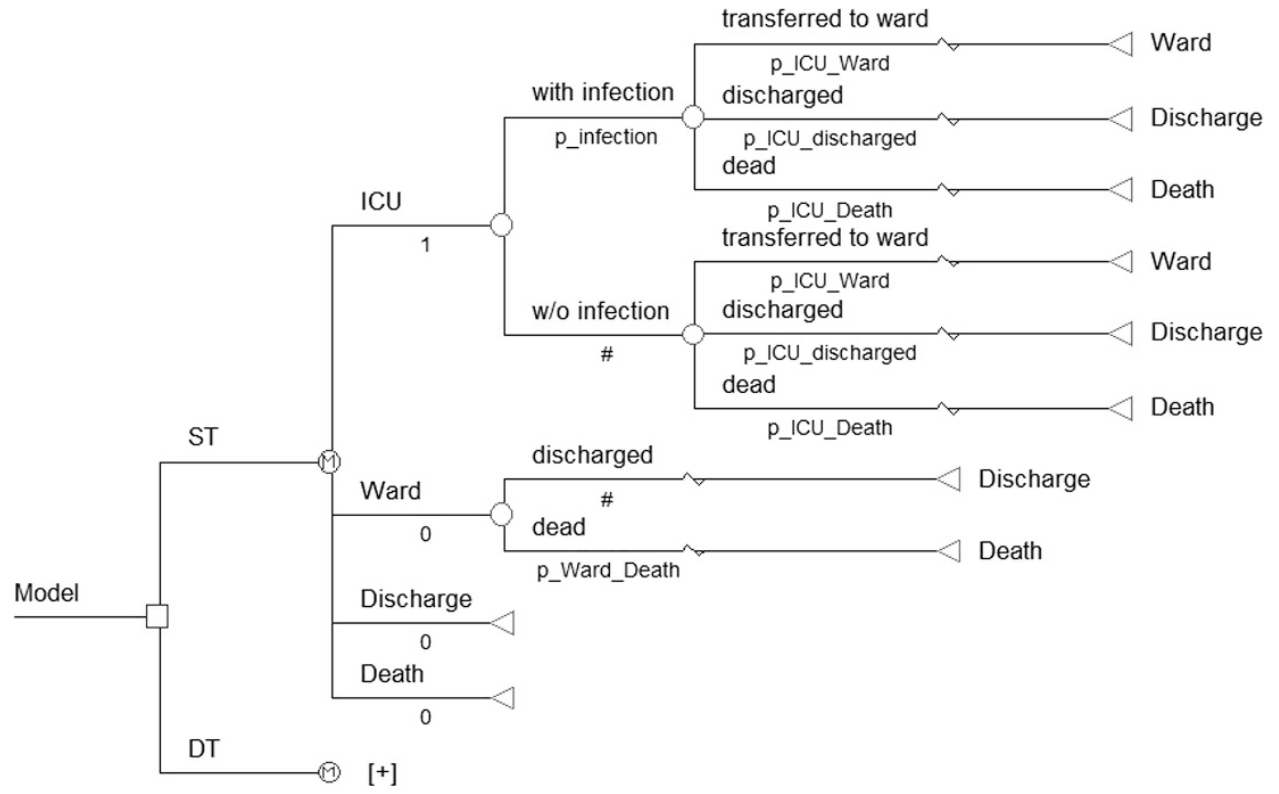

Figure 1. Simplified model structure. 
indirect costs (e.g., working days lost) were considered. Thus, the price of infection, ICU and ward costs were included, with these costs derived from official sources and published literature ${ }^{16-18}$ updated to 2012 values. Adjustment to 2012 values used figures for the Italian consumer price index available from Eurostat. ${ }^{19}$ Thus, the most up-to-date and publicly available figures for Italian health care were used for the daily cost of ICU stay, hospital ward stay and cost of new infections, as follows. The daily cost of ICU stay (€1581.76) was estimated using the results of an Italian 2year prospective case-control study, ${ }^{16}$ subtracting the cost of surgical procedures and cost of extra antibiotics in sepsis patients (which were considered separately for patients developing nosocomial infections in the simulation). The cost for an average day in an Italian hospital ward $(€ 793.30)$ was taken from the report of the National Agency for Regional Health Services (ASSR). ${ }^{18}$ The cost of new infections was included in the cost input for the model based on average prices reported for the treatment of ICU-emergent bloodstream infections. ${ }^{17}$ These costs were limited to those for extra anti-infective treatments needed (i.e., €1114.87) rather than effects of new infections on length of hospital stay or mortality rates, as length of stay and mortality rates were already included in the model as clinical outcomes.

The cost of i.v. glutamine supplementation was calculated per simulated patient on the basis of their body weight and PN duration, assuming a dose of alanyl-glutamine of $0.5 \mathrm{~g} / \mathrm{kg}$ per day, and a price of $€ 2.15 / \mathrm{g}$, which was derived from $50 \%$ of the wholesale price (average price to Italian hospitals) of $€ 85.97$ for a 100 - $\mathrm{ml}$ vial of alanyl-glutamine (Dipeptiven Fresenius Kabi Deutschland GmbH, Bad Homburg, Germany). Supplementation cost was calculated for each simulated patient on the basis of (i) the duration of PN (mean 6.13 days; s.d. 4.77) sampled from a Weibul distribution fitted on Margherita data for ICU patients, and (ii) individual patients' weights in the model (mean $70.55 \mathrm{~kg}$; s.d. 13.39) sampled from a gamma distribution reflecting the populations enrolled in the clinical trials used to determine treatment effectiveness estimates.

\section{Simulation and sensitivity analyses}

The model accounts for variability among individuals (as it is a patient-level simulation), and also the uncertainty of key parameters (by performing a probabilistic sensitivity analysis). It was performed using a two-level Monte Carlo simulation: the inner loop (10000 iterations) was the patient-level

Table 1. Probabilities of outcomes and mean $\operatorname{LOS}^{9}$

\begin{tabular}{lc}
\hline Description & Value \\
\hline Probability of being transferred from the ICU to a general ward & $79.7 \%$ \\
Probability of being discharged directly from the ICU to home & $1.3 \%$ \\
Probability of dying in the ICU & $19.0 \%$ \\
Probability of dying in a general ward & $7.9 \%$ \\
Probability of new infection acquired in the ICU & $11.4 \%$ \\
LOS, pre-ICU, days (s.d.) & $5.60(9.91)$ \\
LOS ICU-alive patients, days (s.d.) & $6.10(11.40)$ \\
LOS ICU—dead patients, days (s.d.) & $8.80(16.04)$ \\
LOS, post-ICU-alive patients, days (s.d.) & $23.20(23.70)$ \\
LOS, post-ICU_dead patients, days (s.d.) & $18.10(24.10)$
\end{tabular}

Abbreviations: ICU, intensive care unit; LOS, length of stay; s.d., standard deviation. Note: To mathematically represent LOS variables in the model, a Weibull distribution was fitted to these data sets, yielding a satisfying 'goodness of fit'. Other distributions were used appropriately according to the type of data (Dirichlet distributions for probability of transfer from ICU to general ward, discharge and death in the ICU; beta distributions for probability of death in a general ward and of new infection in the ICU). simulation, which was averaged and repeated 1000 times (outer loop) each with a unique set of key model parameters drawn randomly from distributions representing the range of plausible values. A standard deviation of $20 \%$ of mean values was supposed for parameters without 95\% Cls (i.e., unit costs, infection probability and glutamine dose). A deterministic (one-way) sensitivity analysis was also used, which tested the sensitivity of estimated money savings per patient to extreme variations of base-case estimates. The 2.5th and 97.5th percentiles of the distributions used in the outer loop of the simulation were used as minimum and maximum values, whereas patient-level variables varied by shifting distributions to the left and right by $20 \%$ of the mean values.

\section{RESULTS}

Main results

PN regimens supplemented with glutamine offer the following advantages over standard PN: they prevent $29.0 \%$ of deaths ( 753 of 2598 ) and $21.2 \%$ of infections (247 of 1165), and they reduce the mean overall length of hospital stay by 1.07 days per patient (Table 3). Glutamine, in the form of i.v. alanyl-glutamine supplementation, is also expected to reduce overall costs compared with standard PN, with a mean net cost saving of $€ 1047$ per patient (Table 3). These results indicate that alanylglutamine costs $(€ 369)$ are more than offset by savings in hospital stay costs (€1388), and also by reduced antibiotic costs for the treatment of ICU-emergent infections $(€ 28)$. Consequently, alanylglutamine is expected to be a more cost-effective option than standard PN, as it is associated with a lower mean cost per patient discharged alive (Table 3). Indeed, on average, it is expected to dominate standard PN, as it is associated with better clinical and economic outcomes.

\section{Sensitivity analyses results}

The probabilistic sensitivity analysis confirmed the robustness of the model results: a strategy of parenteral glutamine supplementation was dominant in $91.2 \%$ of cases (Figure 2). Results of the deterministic (one-way) sensitivity analysis are displayed as a tornado diagram showing the influence of extreme variations in each key parameter on cost savings per patient (Figure 3). This shows that the most influential parameters were the average daily cost of the ICU, cost of parenteral glutamine supplementation, length of stay in the ICU and ICU mortality. None of the variations tested resulted in parenteral glutamine supplementation losing its dominance over standard PN regimens, finding the study to be robust and reliable - that on average $\mathrm{PN}$ regimens supplemented with glutamine are cost saving compared with standard PN regimens.

\section{DISCUSSION}

The results of the analyses presented here suggest that parenteral glutamine supplementation has the potential to significantly improve outcomes for ICU patients in Italy, as our model shows that the additional cost of supplemented treatment is more than completely offset by cost savings in hospital care. Thus, parenteral glutamine supplementation was more cost-effective

Table 2. Clinical outcomes arising from the meta-analysis ${ }^{5}$

\begin{tabular}{|c|c|c|c|}
\hline & Parameter $^{\mathrm{a}}$ & $95 \% \mathrm{Cl}$ & No. of trials (patients) \\
\hline Mortality rate, $\mathrm{RR}$ & 0.69 & $0.54-0.88$ & $16(n=1338)$ \\
\hline Infection rate, RR & 0.79 & $0.67-0.93$ & $19(n=1102)$ \\
\hline
\end{tabular}


(CER $=€ 35165$ per patient discharged alive) than standard, nonsupplemented PN (CER=€40156 per patient discharged alive), and it resulted in mean cost savings of $€ 4991$ per patient discharged alive or $€ 1047$ per patient admitted to the hospital. As supplementation costs were more than completely offset by cost savings to the hospital, glutamine supplementation is said to 'dominate' standard, non-supplemented PN, as it provides better quality of health care for less cost.

The conclusions for the current study are similar to those published previously, but the availability of more specific metaanalysis results have allowed improvements to the clinical sources used in the model to greater reflect best clinical practice in administering $\mathrm{PN}^{6}{ }^{6}$ This gives greater confidence in the applicability and clinical usefulness of the results. This update also relies on a more up-to-date and wider group of ICU patients than our previous study (i.e., not just patients admitted to the ICU for 'intensive care therapies' as in the previous study, but also including less critically ill patients such as those admitted for 'monitoring and weaning', which represent about one-third of all ICU patients). Thus, the current analysis may reflect recent Italian patient populations in ICUs more closely. The current updated pharmacoeconomic analysis uses a longer length of hospital stay, resulting in somewhat higher costs per patient for both standard and glutamine-supplemented PN than in the previous study. Moreover, there are slight differences between the two studies in reduction of infectious complications and mortality rates. Taken together, these differences translate into variations in the economic analysis (total mean net savings per patient of $€ 752$ vs $€ 1047$, for the 2012 study and in this update, respectively). ${ }^{6}$

The stability of the model results has also been demonstrated by both types of sensitivity analysis performed, suggesting that these findings are likely to be applicable to other health-care settings and systems outside Italy-provided that there are no major differences in the ICU patient population, and/or the organisation and costs of the ICU or more generally in tertiary care. Thus, the addition of alanyl-glutamine to standard PN regimens for ICU patients is likely to be very cost-effective (if not dominant) in most European countries. Nevertheless, increased acquisition costs still form a barrier to more widespread use of alanyl-glutamine supplementation of PN.

Several clinical studies have shown that addition of alanylglutamine to nutrition regimens can minimise resource healthcare use. A randomised double-blind study comparing the use of supplemental alanyl-glutamine with standard PN regimens in

Table 3. Outcomes and cost results

\begin{tabular}{|c|c|c|c|c|c|c|}
\hline & Mean & s.e. & Mean & s.e. & Mean & s.e. \\
\hline Deaths per 10000 patients & 2598 & 406 & 1845 & 377 & 753 & 250 \\
\hline Infections per 10000 patients & 1165 & 243 & 918 & 209 & 247 & 91 \\
\hline Total cost (€) & 29724 & 4218 & 28677 & 4066 & 1047 & 823 \\
\hline Glutamine cost $(€)$ & 0 & 0 & 369 & 101 & -369 & 101 \\
\hline CER ( $€ /$ patient discharged alive) & 40156 & 5969 & 35165 & 5181 & 4991 & 1534 \\
\hline
\end{tabular}

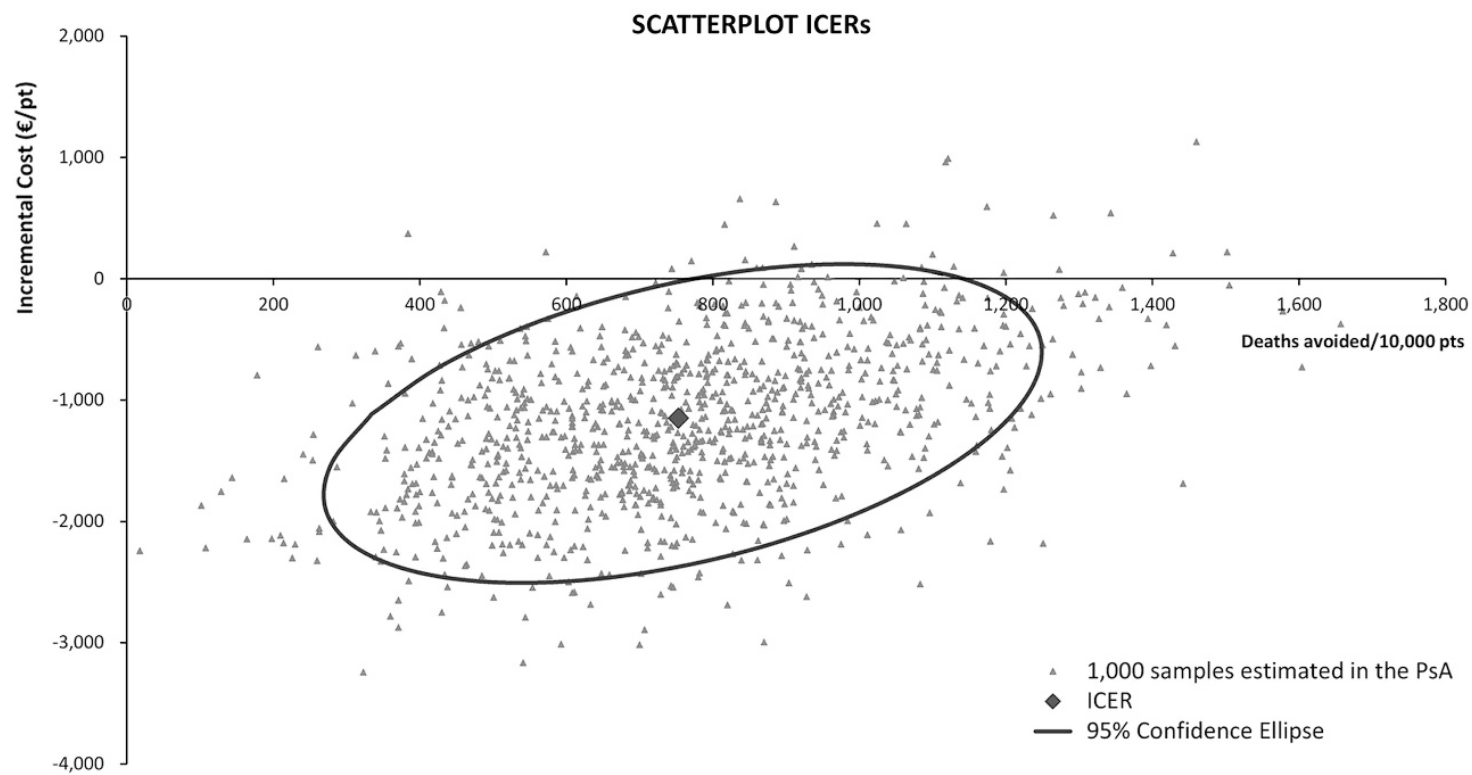

Figure 2. Graphical representation of cost-effectiveness probabilistic sensitivity analysis. This graph shows 1000 samples resulting from the probabilistic sensitivity analysis (PSA) and $95 \%$ confidence ellipse. The density of the 'cloud', around the base-case result, indicates the stability of the model results. pts, patients. 


\section{TORNADO - Saving (Standard vs Dipeptiven ${ }^{\circledR}$ treatment)}

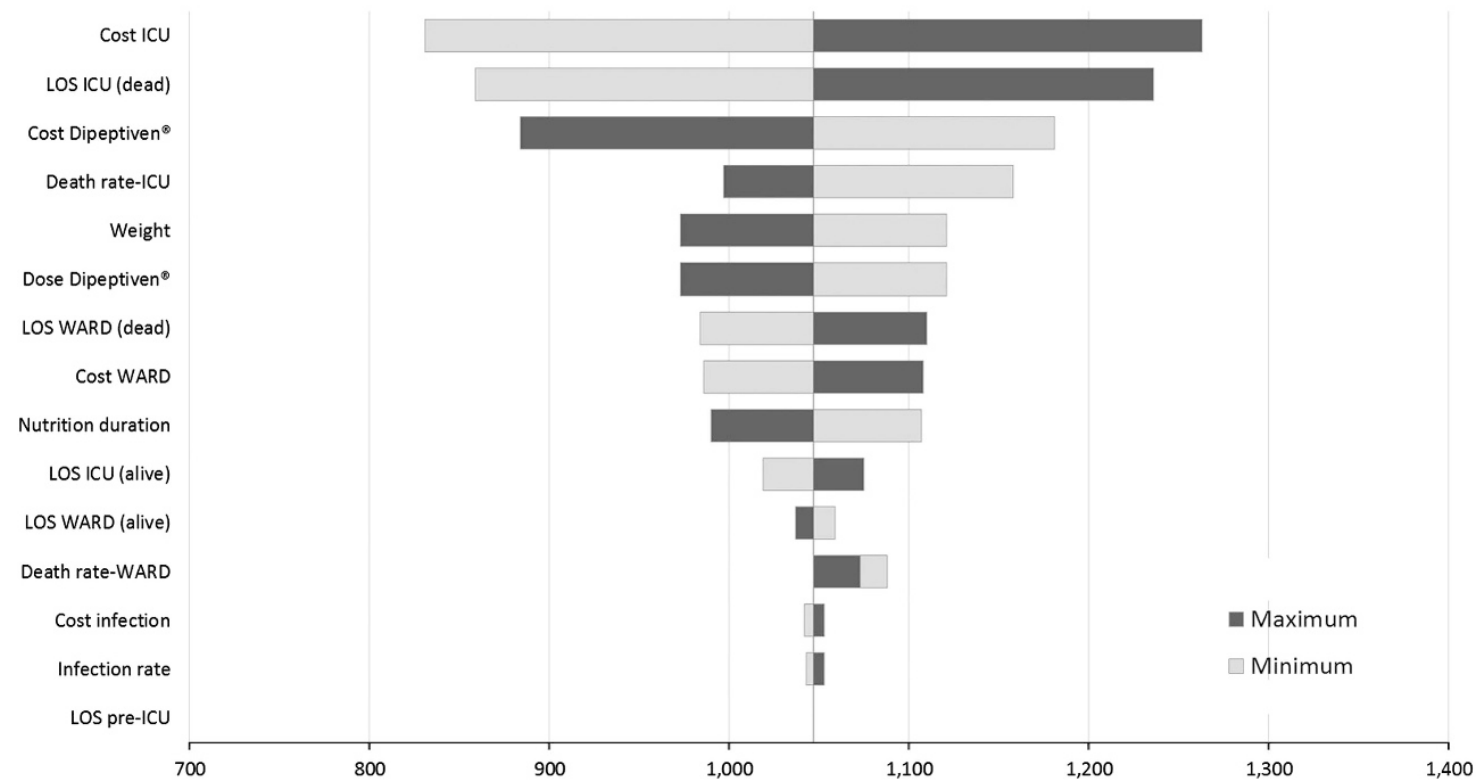

Figure 3. Tornado diagram for deterministic sensitivity analyses: money savings for patients given parenteral glutamine supplementation (PGS).

patients following major abdominal surgery showed that those given alanyl-glutamine had a significantly reduced hospital stay, and thus this strategy allowed potential cost savings. ${ }^{20}$ Another randomised double-blind study in patients with burns found that supplemental alanyl-glutamine significantly reduced wound healing times and hospital costs compared with standard PN. ${ }^{21}$

It is important, however, to understand that we do not suggest that supplementation of all patients' standard PN regimens with alanyl-glutamine will be a cost-effective strategy. It is unlikely to be effective if it is used in an inappropriate clinical scenario: clinicians' discernment and clinical judgment are necessary for the selection of patients in whom supplementation will be beneficial. Alanyl-glutamine is indicated as part of a clinical nutrition regimen in patients in hypercatabolic and/or hypermetabolic states, and it should be given together with parenteral or enteral nutrition or a combination of both. Although glutamine is normally a nonessential amino acid in healthy individuals, it can become conditionally essential under hypercatabolic and hypermetabolic conditions. Thus, patients who are most likely to benefit from i.v. glutamine supplementation include critically ill/ICU patients, those with acute pancreatitis, severe burns, sepsis or following major surgical procedures (e.g., abdominal surgery). ${ }^{5,22-24}$

There are a number of well-known limitations to the modelling of cost-effectiveness: these are intrinsic to modelling itself, and they centre on the need for combining various data sources into a single logical construct. However, the use of modelling in economic evaluations is regarded widely as an 'unavoidable fact of life'. ${ }^{25}$ Models are often used in health economic evaluations, as relevant clinical trials have not been performed or they did not report economic data. Decision analytic models are often used as a tool for health-care decision-makers, allowing the synthesis of the best available data rather than waiting for the perfect set of clinical and economic data to become available. ${ }^{25}$

\section{CONCLUSIONS}

The results of this modelling study strongly suggest that the addition of i.v. glutamine (in the form of alanyl-glutamine) to standard PN is a clinically and economically attractive strategy for ICU patients in Italy. Moreover, the robustness of these results mean that they may be applicable to selected ICU patient populations in other countries.

\section{CONFLICT OF INTEREST}

LP is co-owner and employee of AdRes, which has received project funding from Fresenius Kabi. MP is employee of AdRes, which has received project funding from Fresenius Kabi; MM has received speaker honoraria from Baxter, B Braun and Fresenius Kabi; and ME declares no conflict of interest.

\section{ACKNOWLEDGEMENTS}

We thank Dr Richard Clark and Dr Martina Sintzel for providing writing and editorial assistance on behalf of Fresenius Kabi Deutschland $\mathrm{GmbH}$. Fresenius also made an unrestricted educational grant to meet the cost of preparing this article.

\section{REFERENCES}

1 Oudemans-van Straaten HM, Bosman RJ, Treskes $M$, van der Spoel HJ, Zandstra DF. Plasma glutamine depletion and patient outcome in acute ICU admissions. Intens Care Med 2001; 27: 84-90.

2 Rodas PC, Rooyackers O, Hebert C, Norberg A, Wernerman J. Glutamine and glutathione at ICU admission in relation to outcome. Clin Sci (Lond) 2012; 122: 591-597.

3 Dipeptiven (L-alanyl-L-glutamine). Summary of Product Characteristics. Fresenius Kabi, Bad Homburg, 2011.

4 Albers S, Wernerman J, Stehle P, Vinnars E, Furst P. Availability of amino acids supplied intravenously in healthy man as synthetic dipeptides: kinetic evaluation of L-alanyl-L-glutamine and glycyl-L-tyrosine. Clin Sci (Lond) 1988; 75: 463-468.

5 Bollhalder L, Pfeil AM, Tomonaga Y, Schwenkglenks M. A systematic literature review and meta-analysis of randomized clinical trials of parenteral glutamine supplementation. Clin Nutr 2012; 32: 213-223.

6 Pradelli I, lannazzo S, Zaniolo O, Muscaritoli M, Eandi M. Effectiveness and cost-effectiveness of supplemental glutamine dipeptide in total PN therapy for critically ill patients: a discrete event simulation model based on Italian data. Int J Technol Assess Health Care 2012; 28: 22-28.

7 Singer P, Berger MM, Van den Berghe G, Biolo G, Calder P, Forbes A et al. ESPEN guidelines on PN: intensive care. Clin Nutr 2009; 28: 387-400.

8 McClave SA, Martindale RG, Vanek VW, McCarthy M, Roberts P, Taylor B et al. Guidelines for the provision and assessment of nutrition support therapy in the adult critically ill patient: Society of Critical Care Medicine (SCCM) and American Society for Parenteral and Enteral Nutrition (A.S.P.E.N.). J Parenter Enteral Nutr 2009; 33: 277-316 
9 Progetto MARGHERITA-Rapporto 2010. Gruppo Italiano per la Valutazione degli Interventi in Terapia Intensiva (GIVITI). Sestante Edizioni: Bergamo, 2011.

10 Progetto MARGHERITA-Rapporto 2007. Gruppo Italiano per la Valutazione degli Interventi in Terapia Intensiva (GIVITI). Sestante Edizioni: Bergamo, 2008.

11 Malacarne $P$, Rossi C, Bertolini G. Antibiotic usage in intensive care units: a pharmaco-epidemiological multicentre study. J Antimicrob Chemother 2004; 54: 221-224.

12 Boffelli S, Rossi C, Anghileri A, Giardino M, Carnevale L, Messina M et al. Continuous quality improvement in intensive care medicine: the GiViTI Margherita project-report 2005. Miner Anestesiol 2006; 72: 419-432.

13 Galzerano A, Sabatini E, Duri D. Old patients in intensive care unit (ICU): What decisions to make? Arch Gerontol Geriatr 2009; 49: 294-297.

14 Poole D, Rossi C, Anghileri A, Giardino M, Latronico N, Radrizzani D et al. External validation of the Simplified Acute Physiology Score (SAPS) 3 in a cohort of 28,357 patients from 147 Italian intensive care units. Intens Care Med 2009; 35: 1916-1924.

15 Bertolini G, Boffelli S, Malacarne P, Peta M, Marchesi M, Barbisan C et al. End-of-life decision-making and quality of ICU performance: an observational study in 84 Italian units. Intens Care Med 2010; 36: 1495-1504.

16 Tarricone R, Torbica A, Franzetti F, Rosenthal VD. Hospital costs of central line-associated bloodstream infections and cost-effectiveness of closed vs open infusion containers. The case of intensive care units in Italy. Cost Eff Resour Alloc 2010; 8: 8.

17 Orsi GB, Di Stefano L, Noah N. Hospital-acquired, laboratory-confirmed bloodstream infection: increased hospital stay and direct costs. Infect Control Hosp Epidemiol 2002; 23: 190-197.

18 Agenzia Nazionale per i Servizi Sanitari Regionali (ASSR). Ricoveri, personale e spesa delle aziende ospedaliere (2003) http://www.agenas.it/images/agenas/ monitor/pdf/Monitor_16.pdf (last access November 2013).

19 European Commission. EuroStat 2012: http://epp.eurostat.ec.europa.eu/portal/ page/portal/hicp/data/database (last access November 2013).
20 Mertes N, Schulzki C, Goeters C, Winde G, Benzing S, Kuhn KS et al. Cost containment through L-alanyl-L-glutamine supplemented total PN after major abdominal surgery: a prospective randomized double-blind controlled study. Clin Nutr 2000; 19: 395-401.

21 Zhou Y-P, Jiang Z-M, Sun Y-H, He G-Z, Shu H. The effects of supplemental glutamine dipeptide on gut integrity and clinical outcome after major escharectomy in severe burns: a randomized, double-blind, controlled clinical trial. Clin Nutr Suppl 2004; 1: 55-60.

22 Wang $Y$, Jiang Z-M, Nolan MT, Jiang H, Han HR, Yu K et al. The impact of glutamine dipeptide-supplemented parenteral nutrition on outcomes of surgical patients: a meta-analysis of randomized clinical trials. J Parenter Enteral Nutr 2010; 34: 521-529.

23 Zheng Y-M, Li F, Zhang M-M, Wu X-T. Glutamine dipeptide for parenteral nutrition in abdominal surgery: a meta-analysis of randomized controlled trials. World J Gastroenterol 2006; 12: 7537-7541.

24 Novak F, Heyland DK, Avenell A, Drover JW, Su X. Glutamine supplementation in serious illness: a systematic review of the evidence. Crit Care Med 2002; 30: 2022-2029.

25 Buxton MJ, Drummond MF, Van Hout BA, Prince RL, Sheldon TA, Szucs T et al. Modelling in economic evaluation: an unavoidable fact of life. Health Econ 1997; 6: 217-227.

(2) This work is licensed under a Creative Commons Attributioncc. No SA SA Nommercial-ShareAlike 4.0 International License. The images or other third party material in this article are included in the article's Creative Commons license, unless indicated otherwise in the credit line; if the material is not included under the Creative Commons license, users will need to obtain permission from the license holder to reproduce the material. To view a copy of this license, visit http:// creativecommons.org/licenses/by-nc-sa/4.0/ 